\title{
ESTUDIOS SOBRE MODELOS DE CONSUMO: UNA VISIÓN DESDE TEORÍAS Y METODOLOGÍAS
}

\author{
STUDIES ABOUT ALIMENTARY MODELS: \\ A VIEW FROM THEORIES AND METHODOLOGIES
}

\author{
Maria Viola T. \\ Centro de Estudios del Sistema Agroalimentario, \\ Universidad Nacional de Lomas de Zamora, Buenos Aires, Argentina.
}

\begin{abstract}
In each society, feeding is constructed from a symbolic process that characterizes foods like edible, inserts them in a system of preferences and establishes the forms of preparation and consumption. The description, explanation and prediction of the alimentary behavior present an increasing complexity on the basis of the appearance of new manifestations about well-known factors and new phenomena to know. This article will show the influence of the identity, the relation between supply and consumption, the conditioners of the intake, the subject, the life style, the sensorial dimensions of foods and body discourses for discussing its methodological treatment.
\end{abstract}

Key words: alimentary behavior, methodology, research techniques

Este trabajo fue recibido el 1 de Marzo de 2007, aceptado con modificaciones el 6 de Junio de 2007 y aceptado para ser publicado el 10 de Mayo de 2008.

\section{INTRODUCCIÓN}

En cada sociedad, la alimentación se construye a partir de un proceso de simbolización que caracteriza a los alimentos como comestibles, los inserta en un sistema de preferencias y les establece las formas de preparación y su ocasión de consumo. Las preferencias por ciertos alimentos en desmedro de otros se presentan como rechazos (neofobias), temor a padecer enfermedades, asociación con experiencias agradables o desagradables vinculadas a ciertos alimentos, creencias, prejuicios, imitación, disponibilidad, poder adquisitivo, nivel socioeconómico, innovación, religión y cultura culinaria (1).

La complejidad del acto de comer hace necesario recurrir a métodos que escapan de las ciencias biológicas para poder interpretar los hábitos dietéticos superando la mera información cuantitativa de qué y cuánto se come para lograr una aproximación que permita saber porqué se come. En ese dominio, los discursos de ingesta, la oferta, la demanda y el ambiente cultural organizan/reorganizan los modos de articulación con el territorio y las ideas circulantes en torno al cuerpo, a la dieta y a la salud configurando modelos de consumo derivados de una práctica heredada tanto como de circunstancias locales.

Si bien los méritos de aproximaciones subjetivas contra las objetivas en el estudio de los comportamientos han sido debatidos (2) ambas son necesarias para comprender el consumo. Las aproximaciones cuantitativas son representaciones estadísticas sobre variables continuas o discretas que entregan información sobre actitudes alimentarias específicas. 
Las indagaciones cualitativas ponen énfasis en el comportamiento, los hábitos y los procedimientos en orden a factores de contexto que los condicionan. La integración de ambas posiciones de análisis conduce a una interpretación más precisa de la comensalidad en la medida que tomen en cuenta la incidencia de una serie elementos que afectan los modos de estructuración de la matriz de datos del patrón alimentario. En efecto, las comidas, los platos y los alimentos se eligen, preparan y sirven según un orden contextual complejo donde algunas reglas atienden a la exclusión y otras a la inclusión(3).

El objetivo de este artículo es revisar un conjunto de aspectos relacionados con el comportamiento alimentario que aportan a la mejor comprensión del modelo de consumo, discutirlos y procurar establecer vínculos con el método.

\section{EL CONTEXTO DE LA ALIMENTACIÓN}

El proceso de alimentación se cumple en el seno de una comunidad en la que están disponibles sólo ciertos ingredientes: La consolidación, la desaparición o la transformación de componentes de la estructura alimenticia proceden de una múltiple conexión con los procesos económico productivos, comerciales y sociales.

Para comprender los modos de alimentarse de una población es necesario: 1. Establecer los rasgos de la oferta de alimentos, 2. Comprender las prácticas de selección (desde las imágenes previas, positivas o negativas, del alimento hasta los modos de operar del consumidor), 3. Reconocer las técnicas disponibles de preparación y cocción (aplicaciones de los ingredientes), 4. Comprender las reglas de la comensalid ad (donde las construcciones sociales de la ingesta ubican/reubican a los ingredientes) y 5. Comprender las influencias de la oferta en los atributos de los ingredientes (formas de presentación, distribución, conservación y almacenamiento).

Los términos del diseño metodológico varían de acuerdo a la orientación de la investigación (cultural, social, psicológica, fisiológico, nutricional, económica, entre otras), el nivel del análisis (un tipo de alimento, repertorio de alimentos consumidos, modos de consumo, entre otros), y el tipo de abordaje de la investigación (diagnóstico de problemas, descriptivo, explicativo o predictivo). En todos ellos el investigador debe considerar las implicancias de algunos temas actuales para interpretar sus resultados. Estos temas son: influencia de la identidad, relación entre oferta y consumo, condicionantes de la ingesta, sujeto, estilo de vida, dimensión sensoria de los alimentos y discursos sobre el cuerpo.

\section{LA INFLUENCIA DE LA IDENTIDAD}

El proceso de globalización de los mercados de alimentos impulsa a considerar una aparente homogeneidad de la población en materia de selección alimentaria. La identidad, como conjunto de preferencias de un grupo social determinado, ha sido señalada como un móvil de la conducta en materia de las selecciones alimentarias, sosteniéndose que los principios de selección que guían al ser humano en la elección de sus recursos alimentarios no son, según todas las apariencias, de orden fisiológico, sino cultural (4).

Asimismo, la alimentación como elemento identificador de un grupo social, es la característica que más difícilmente se pierde en el contacto con otros grupos y la perpetuación del estilo alimentario original en la vida cotidiana es una prueba de autenticidad y de cohesión social y una defensa contra las agresiones externas $(5,6)$.

La aplicación rigurosa de las reglas alimentarias constituye una protección contra la aculturación y la pérdida de identidad en los contactos interculturales y en consecuencia, es lógico que los colectivos de inmigrantes no quieran abandonar ciertas prácticas alimentarias, sino todo lo contrario, crean en la sociedad de recepción espacios donde poder satisfacer este tipo de peculiaridades $(7,8)$. 
La consecuencia tanto de los fenómenos migratorios como de nuevos modos de acreditar propiedades (certificación, denominaciones de origen, entre otros), promueve que en una misma región, se desarrollen movimientos de interculturalidad alimentaria. Estos configuran nuevas interacciones sociales que inciden los tratamientos de los resultados de las investigaciones. Comer "como nosotros" o comer como "los otros" puede implicar un proceso de legitimación por un lado y un proceso de competitividad por el otro, confluyendo sin embargo durante el análisis en un proceso aparentemente equivalente.

En el sentido metodológico la estructura alimentaria de las poblaciones requiere de tres movimientos iniciales de indagación: la etnografía, la historia de vida y la observación directa o participante de los comportamientos. La articulación de estos tres tipos de indagaciones permite seguidamente la discriminación en dos niveles: el de la dieta como acto de ingesta pleno de significados y el de la alimentación como articulación de una lógica de prácticas y preferencias especificadas a partir de variables de parentesco, origen étnico y datos sociodemográficos de los sujetos involucrados.

\section{RELACIÓN OFERTA-CONSUMO}

De acuerdo con algunos autores, las actividades de producción, transformación industrial y comercio agroalimentario, no pueden ser examinadas de manera parcial y aislada, sino que deben ser concebidas como un sistema caracterizado por las interrelaciones entre la oferta agrícola, el acopio, el almacenamiento, el procesamiento, la distribución y el consumo, resaltando los procesos de integración vertical y horizontal entre la agricultura y la industria transformadora de las materias primas agrícolas (9).

A finales de la década de 1960, se desarrolló un marco teórico-metodológico de la economía agroalimentaria, mediante una serie de trabajos teóricos y empíricos (10). Como resultado, se definió el sistema agroalimentario como un conjunto de actividades económicas interrelacionadas con actores que participan en la producción, transformación y distribución de alimentos con la finalidad de satisfacer las necesidades de energía y nutrientes de la población, tomando en cuenta la calidad e inocuidad de los mismos para cumplir satisfactoriamente con la función social alimentación-nutrición. Esta visión plantea que desde un punto de vista funcional, existen una serie de asociaciones entre alimentación y entorno económico que evoluciona según se modifican los medios, tanto como los modos de producción y distribución. Los alimentos orgánicos o genéticamente modificados, por ejemplo, sobre los que las posiciones de los consumidores se presentan complejas y difíciles de ponderar, constituyen estrategias de producción que definen nuevos productos. Por otro lado, a pesar de la valoración otorgada actualmente a la publicidad, el cambio en la alimentación se produce a partir de mecanismos más articulados y complejos en los que influyen: crisis crónicas o episódicas en la disponibilidad de alimentos (vaca loca, dioxinas), los programas de ayuda alimentaria (en cuanto ponen en contraste las selecciones de la población atendida con la lógica de priorización de los organismos asistenciales), las transformaciones tecnológicas y ecológicas de la producción, la industrialización, la urbanización y las migraciones (11).

Un estudio pormenorizado del consumo debe incluir una revisión previa sobre las características de los productos y sus modos de aprovisionamiento, almacenamiento, conservación, uso de la tecnología y de la energía como base para interpretar los modos de operar de los consumidores.

El estudio de los hábitos alimentarios dentro de los procesos globalización requiere consideraciones metodológicas de cierta especificidad y una de sus finalidades puede consistir en comparar los grados de penetración de tipos de alimentos en la dieta de las poblaciones urbanas o bien de las pautas que el consumidor pone en práctica para el ejercicio de sus aceptaciones o rechazos. 
Para este tipo de análisis conviene recurrir a procesos descriptivos de la oferta que presten apoyo a la construcción de encuestas estructuradas dirigidas a establecer el uso de los productos existentes y las prácticas de consumo que las acompañan.

\section{LOS CONDICIONANTES DE LA INGESTA}

La oferta y la demanda de alimentos no operan en planos de absoluta libertad. Asimismo la oferta aparece como resultado de las prácticas productivas eficientes dentro de una cadena de valor, en tanto que la demanda aparece más ligada a la intensidad de adhesión a los discursos sobre la corporalidad, la nutrición y la salud.

En consecuencia la interpretación del consumo requiere encontrar las posiciones que los consumidores adoptan para ampliar o sustituir alimentos dentro del repertorio preexistente y el tipo de discurso que gobierna sus elecciones. Para este tipo de objetivos los grupos de discusión (12) permiten mejores resultados pero requieren una ardua tarea previa destinada a armar las guías y reclutar convenientemente a los entrevistados.

La configuración de la dieta difiere en cuanto se la considera dentro y fuera del hogar. En este sentido es necesario considerar la tecnología disponible dentro de las cocinas familiares y colectivas para visualizar las estrategias que tienen lugar en cuanto a la conservación, el almacenamiento, la preparación y la cocción. La observación de la oferta en base a los productos disponibles en los puntos de distribución minorista hace posible la correspondencia entre las propiedades de los productos y las preferencias.

\section{EL SUJETO DEL CONSUMO}

Un aporte teórico importante para la consideración del sujeto del consumo señala la existencia de tres principios de la alimentación: la circunstancia paradojal del hombre como omnívoro, el principio de incorporación y la construcción del sí mismo en términos de una identificación(3).

El primer principio alude a una relación de tensión entre h libertad de selección de alimentos y una restricción para cambiar el repertorio. Esta doble vía delimita y explica un espacio rígido unido a un espacio de mutación en el que los procesos de ampliación del repertorio se cumplen en acuerdo con ciertas reglas de existencia previa que el investigador puede conocer recurriendo a la entrevista en profundidad (13).

El principio de incorporación postula la existencia de una combinación nutricional y simbólica ejercida durante la apropiación de las costumbres alimentarias. La incorporación construye una identidad de cuerpo a la vez que materializa una identidad del ser persona en los términos de un discurso cultural. La delimitación de este discurso cultural puede quedar especificada cuando se describen los platos y recetas más comunes a los que recurren los individuos.

El tercer principio toma en cuenta las relaciones del individuo en la esfera de su propio dominio donde se instalan los conjuntos de prácticas básicas conectadas a variaciones surgidas de procesos de innovación y adaptación. Las inclinaciones individuales están alineadas con el acto de comer como proceso íntimo y cotidiano que puede ser visualizado en subconjuntos de hábitos vinculados al comportamiento psicosocial.

Los estudios de consumo requieren dos fases importantes, una que atañe a las relaciones positivas o de aceptación de propiedades de los alimentos y otra, negativa o de rechazo sobre esas mismas atribuciones. Estos planos de la indagación son posibles con el uso de las técnicas de observación participante, entrevistas en profundidad y grupos de discusión, en la etapa cualitativa; y de encuestas, en la cuantitativa, donde la unidad de análisis más aconsejable es la que remite a familias u hogares, aunque estos sean unipersonales.

Por otro lado, en el terreno de la estructura de la alimentación de un grupo social, el sujeto se despliega ejerciendo algunos actos y delegando otros. Como consecuencia algunos 
ingredientes son principales y otros se comportan como adicionales y conllevan reglas que les otorgan entidad y relevancia para las combinaciones dentro de un menú conforme el momento del día y la ocasión de comida. Los sujetos ejercen el mecanismo alimentario a través de tres "composiciones": una relativa a los ingredientes, otra a los platos y una tercera a los menús.

El diseño de una encuesta alimentaria requiere discriminar los modos de operar en orden a estos tipos de composiciones. En un estudio reciente una aproximación cualitativa permitió caracterizar los episodios de comida, identificar los temas culturales y los procesos sociales que los caracterizaron y examinar cómo los individuos repiten conductas.

La penetración en la multidimensionalidad del comer y del beber de este trabajo arroja una serie de implicancia tanto para la sociología alimentaria como para los profesionales de la salud y los nutricionistas. Primero las personas percibieron más a menudo el tipo de episodio en el que comen o beben que el alimento o bebida consumida. En segundo lugar se detectaron un gran número de influencias detrás de las opciones por ciertos alimentos. Tercero, las formas de denominar los episodios de ingesta demostraron la prevalencia de una perspectiva personal más que un tipo de perspectiva externa surgida de la ciencia de la nutrición (14). Estos hallazgos conducen a la necesidad de indagaciones cualitativas anteriores al diseño de encuestas, considerar la incidencia de la memoria en las respuestas e incluir preguntas que describan el peso de factores que influyen la ingesta.

Existen evidencias (15) de que los modos de alimentarse a nivel individual contienen un conjunto de inercias que hacen predecible el modelo de consumo de algunos alimentos o bien que la operación de cambios requieren otros vinculados con la estructura alimentaria del grupo de convivencia. Este hecho es de particular interés tanto para objetivos comerciales como para los nutricionistas.

\section{DIMENSIÓN SENSORIA DE LOS ALIMENTOS}

Es necesario considerar un enfoque, aunque complejo de ponderar, donde la selección del alimento es gobernada por un número de características tales como la textura, el olor, el color (además de otras características visuales) incluso el sonido (crocancia); con los que se conjugan aspectos fisiológicos percibidos en la saciedad o en la digestibilidad. Se trata de la dimensión sensorial del alimento. También pueden incluirse los patrones para condimentar, la dulcificación y la salazón que permiten interpretaciones que les otorgan carácter de marcadores de la clase social. En la cultura americana, los alimentos muy dulces son interpretados como fenómeno de clase baja o extranjero; también se ha sugerido que hay una oposición simbólica general entre los alimentos salados, en cuanto a "blando, quebradizo, enérgico, y agudo" y "alimentos dulces" en culturas occidentales (16).

El tamaño y la forma son características sensoriales adicionales de los alimentos que influencian la selección. Estas propiedades aspectuales codifican a menudo la información previa y, asociados a la textura, permiten un juicio sobre el carácter "apetitoso" que inscribe otro punto importante sobre la valoración de un alimento y que pone en juego la dimensión placentera de la comida en oposición a un gobierno estricto de la ingesta. Las dimensiones sensoriales de los alimentos pueden explicar diferencias entre las preferencias de poblaciones rurales y urbanas, diferencias de género o por inadecuación a ciertos miembros de la población que surgen de variadas fuentes informativas donde operan los discursos míticos o los discursos científicos.

Las comparaciones entre grupos humanos basadas en la dimensión sensoria sirven para la traducción de un "gusto cultural" determinado. Metodológicamente se logran descripciones diferenciadas aceptables cuando se vinculan variables de comportamiento selectivo con los rasgos de los sujetos y se analizan en base a métodos estadísticos multivariantes. Los alimentos pueden ser sujetos a clasificaciones basadas en sus propiedades y cómo éstas inciden en las preferencias según los medios de comunicación que inciden en su difusión. En 
estos casos es necesario incorporar elementos de semiótica que permitan relacionar la influencia de los discursos de autoridad y sistemas de creencias para formular adecuadamente los tipos de variables y sus modos de medición. Estadísticamente los análisis de conglomerados, los análisis factoriales y los modelos discriminantes permiten encontrar una multiplicidad de posibles asociaciones entre hábitos y tipo de alimentos (17).

\section{ESTILO DE VIDA}

Dos de los factores que más han influido en la modificación de los modelos alimentarios son, sin duda, el proceso de urbanización y $\mathbf{b}$ modernización de los estilos de vida. Estos fenómenos repercuten en los hábitos alimentarios de diferentes formas. Principalmente lo hacen con la introducción de nuevos alimentos, la delegación de parte del proceso de preparación, la disminución del uso de productos frescos, la modificación de los lugares de consumo y cambios en los usos del tiempo.

Por otro lado, en la sociedad urbana la mayoría de los consumidores son receptores de una oferta que deriva no sólo de mercados locales sino de otros distantes. Esta situación deriva en una modificación de la tradición culinaria. La existencia de una tradición culinaria de élite deriva de las posiciones de los grupos sociales en elación a la posibilidad de organizar y componer un repertorio alimentario extenso y relevante donde la comida emerge como un plato realizado bajo ciertas normas y mediante ciertas tecnologías donde el despliegue culinario se cumple por la vía del dominio de la distribución (18).

Cuando se realizan encuestas sobre los procesos de alimentación es necesario incluir variables sobre la motivación de ciertos hábitos, el origen de la selección alimentaria, los modos de organización del hogar (en cuanto a aprovisionamiento, estructura ocupacional del grupo, lugar y ocasión de consumo) y las características del grupo social con el que se desarrolla la ingesta.

En estas nuevas situaciones el estudio de alimentos fuertemente locales y sus modos de incorporación permite incursionar en los valores simbólicos que se privilegian dentro de un territorio (urbano o rural) por un determinado grupo social, dentro de cuyos parámetros pueden conducirse las sustituciones o las nuevas incorporaciones. Sin embargo el estudio de los alimentos locales presenta dificultades dado que están sometidos a la práctica artesanal tanto en los modos de adición de valor como en los modos de manipulación y distribución.

Finalmente, los hábitos y actitudes de los consumidores urbanos se inclinan cada vez más a realizar episodios de alimentación fuera del hogar. Con ello aparece la necesidad de considerar la externalización de la alimentación como una variable compleja en la investigación del patrón alimentario y considerar la aparición, en el terreno metodológico, de una nueva unidad de análisis: la gastronomía.

\section{EL DISCURSO DEL CUERPO}

En la organización de la sociedad existen pautas externas al individuo que están relacionadas con la imagen o apariencia de la corporalidad que circulan a través de medios de comunicación. En todos los grupos sociales el cuerpo es objeto de definición y estas se instalan básicamente a partir de las ideas de belleza y salud. Sin ingresar en el terreno de las patologías, puede decirse que todos los discursos sobre la corporalidad se encuentran indisolublemente ligados a los modos de comer y, por lo tanto, a las posiciones de los individuos con las responsabilidades y valores asumidos respecto a la comida y el ayuno, por un lado, y a la variedad de actitudes que asume n frente a los alimentos, por el otro.

En las sociedades modernas circulan permanentemente discursos sobre la bondad de comer determinados alimentos para alcanzar ciertos ideales corporales confrontados con posiciones relacionadas con alimentos cuyas propiedades no toman en cuenta virtudes 
nutricionales sino relacionadas con proporcionar placer. Es en ese amplio recorrido de los discursos sobre la corporalidad derivados de la moda, del alcance de una imagen idealizada, de un estado de salud, donde los individuos restringen o amplían sus repertorios de alimentos con el objeto de intercambiar posiciones del cuerpo propio en relación a las posiciones de un cuerpo de naturaleza social.

Aquí aparecen campos de indagación discursiva tales como "light", "natural", "diet", "sano", "natural", "libre de" que remiten a un recorte de conductas vinculadas al bienestar del cuerpo. Por otro lado, bs trastornos de la alimentación ponen en evidencia que las relaciones entre cuerpo y alimentación están presentes en forma permanente en las conductas selectivas en distintos grados y su discriminación eficaz en los estudios de consumo los coloca en otros campos de análisis más pertinentes del campo de los nutricionistas o de los estudios de salud.

\section{CONCLUSIONES}

La descripción, explicación y predicción del comportamiento alimentario presenta una complejidad creciente en base a la aparición de nuevas manifestaciones de factores ya conocidos y de nuevos fenómenos por conocer. Dado que existen diferentes perspectivas en los estudios alimentarios es necesario delimitar el campo de análisis, explicitar los objetivos en función del diagnóstico de situación del problema y definir correctamente el objeto de estudio y sus unidades de análisis. Asimismo los modelos de consumo y las relaciones que pueden detectarse en ellos, conectados al comportamiento de los grupos sociales, se presentan complejos y las teorías que dan sustento a la selección de variables deben ser convenientemente especificadas.

En el terreno metodológico, los métodos cualitativos y cuantitativos contribuyen para distinguir, por un lado, las características de los universos simbólicos y, por otro lado, los rasgos de las prácticas efectivas que en conjunto, permiten organizar las posiciones de los consumidores. Las problemáticas de los universos simbólicos son mejor abordadas cuando se recurre a la etnografía, la historia de vida, la entrevista en profundidad o la observación directa. En cambio las prácticas y los hábitos, como modos de operar en lo cotidiano, pueden analizarse mediante la observación directa, el grupo de discusión o la encuesta estructurada. La cuestión central de estos dos grupos de técnicas, es cómo se tienden los puentes y complementaciones entre ellas. En ese sentido, las indagaciones cualitativas detectan las variables y las unidades de análisis de la descripción del patrón alimentario; en tanto que las cuantitativas miden la intensidad de ocurrencia de esas variables en la definición de conductas. El manejo de los datos con herramientas estadísticas multivariadas completa la posibilidad de avanzar en objetivos de investigación que permitan explicar y predecir las actitudes de los sujetos, con ciertos rasgos sociodemográficos y bajo ciertas condiciones, para configurar sus comportamientos frente a una oferta de alimentos especificada.

\section{RESUMEN}

En cada sociedad, la alimentación se construye a partir de un proceso de simbolización que caracteriza a los alimentos como comestibles, los inserta en un sistema de preferencias y les establece las formas de preparación y ocasión de consumo. La descripción, explicación y predicción del comportamiento alimentario presenta una complejidad creciente en base a la aparición de nuevas manifestaciones de factores ya conocidos y de nuevos fenómenos por conocer. En este artículo se tratan la influencia de la identidad, la relación entre oferta y consumo, las condicionantes de la ingesta, el sujeto, el estilo de vida, la dimensión sensoria de los alimentos y discursos sobre el cuerpo discutiendo su tratamiento metodológico.

Palabras claves: comportamiento alimentario, metodología, técnicas de investigación. 
Dirigir la correspondencia a:

Ing. Agr. MSc. María Viola T.

Centro de Estudios del Sistema Agroalimentario

Facultad de Ciencias Agrarias

Universidad Nacional de Lomas de Zamora

Ruta 4 Km. 2 (1836) Llavallol

Bueno Aires - Argentina.

Telefax 54-11-4282-6263/7896/7905

E-mail: violaventurino@yahoo.com.ar; viola@agrarias.unlz.edu.ar

\section{BIBLIOGRAFÍA}

1. De Garine I, Vargas L. A. "Introducción a las investigaciones antropológicas sobre alimentación y nutrición". Rev Cuadernos Nutr 1997;20:21-28.

2. Miller, G., \& Holstein, J. A. (Eds.). Constructivist controversies: Issues in social problems. Ed. Hawthorne, New York, 1993.

3. Fischler, C. El (h)omnívoro. El gusto, la cocina y el cuerpo, Ed. Anagrama, Barcelona, 1995.

4. Douglas, M. "Las estructuras de lo culinario". En Contreras, J. (ed.) Alimentación y cultura. Necesidades, gustos y costumbres.: Publicaciones Universidad de Barcelona, Barcelona pp.171, 1995.

5. Bordieu, P., La Distinción. Ed.Taurus, Madrid, 1988.

6 De Garine, I, "Culture et nutrition", Communications, $\mathrm{n}^{\circ} 31$ (La nourriture), pp 70-92, 1979.

7. Medina, F. X., "Alimentación, etnicidad y migración: ser vasco y comer vasco en Cataluña". En Gracia, M. Somos lo que comemos: estudios de alimentación y cultura en España. Ed.Ariel Antropología, Barcelona, pp. 122-147. 2002.

8. Gracia Arnaiz, M. "La alimentación en el umbral del siglo XXI: una agenda para la investigación sociocultural en España”. En Gracia, M. Somos lo que comemos: estudios de alimentación y cultura en España. Ed.Ariel Antropología, Barcelona, . pp. 15-38, 2002.

9. Davis, J. y Goldberg, R., A concept of agribusiness, Harvard Business School, Division of Research, Boston, 1957.

10. Malassis, L. , Ghersi, G. Initiation a l'économie agroalimentaire, Ed. Université de Paris, París, 1992.

11. Kaplan, A. y Carrasco, S. "Cambios y continuidades en torno a la cultura alimentaria en el proceso migratorio: de Gambia a Cataluña, En Gracia, M. Somos lo que comemos: estudios de alimentación y cultura en España, Ed. Ariel Antropología, Barcelona, 2002.

12. Morgan, D., Focus group as qualitative research, Ed. Sage Publications, London, 1997.

13. Valles, M. Técnicas cualitativas de investigación social. Reflexión metodológica y práctica profesional. Ed. Síntesis Sociológica. Madrid, 1997.

14. Bisogni, C., Winter Falk, L., Madore, E., et al., "Dimensions of everyday eating and drinking episodes" Appetite 48 pp. 218-231, 2007.

15. Mejía, M. "Preferencias alimentarias como alternativa al estudio del patrón dietético" Rev Esp Nutr Comunitaria 2002:8;(1-2):29-34.

16. Barthes, R. "Toward a Psychosociology of Contemporary Food Consumption", In: Forster, E. and Forster, R. (eds.), European Diet from Pre-industrial to Modern Times, Harper Torchbooks, New York, 1975.

17. Johnson, R.A. y Wichern, D.W. Applied Multivariate Statistical Analysis. Prentice-Hall, 3ra. Ed. 1992. 
18. Goody, J. Cooking, Cuisine and Class: A Study in Comparative Sociology, Cambridge University Press, 1982. 